\title{
GSTT1 null genotype is a risk factor for diabetic retinopathy in Caucasians with type 2 diabetes, whereas GSTM1 null genotype might confer protection against retinopathy
}

\author{
Ines Cilenšek ${ }^{\mathrm{a}, 1}$, Sara Mankoča,1, Mojca Globočnik Petrovič ${ }^{\mathrm{b}}$ and Daniel Petroviča,* \\ ${ }^{a}$ Institute of Histology and Embryology, Medical Faculty Ljubljana, University of Ljubljana, Ljubljana, Slovenia \\ ${ }^{\mathrm{b}}$ Eye Clinic, University Medical Centre Ljubljana, Ljubljana, Slovenia
}

\begin{abstract}
Aim: Substantial data indicate that oxidative stress is involved in the development of diabetic retinopathy (DR). The aim of the present study was to investigate whether the genetic polymorphisms: polymorphic deletions of glutathione S-transferases M1 (GSTM1) and T1 (GSTT1) and Ile105Val of the GSTP1 are associated with DR in Slovenian patients with type 2 diabetes. Methods: In this cross sectional case-control study 604 unrelated Slovene subjects (Caucasians) with type 2 diabetes mellitus were enrolled: 284 patients with DR (cases) and the control group of 320 subjects with type 2 diabetes of more than 10 years' duration who had no clinical signs of DR. Genotypes were determined by polymerase chain reaction (PCR) and restriction fragment length polymorphism (RFLP).

Results: In our study, the deletion of the GSTM1 was found less frequent in cases with DR than in the controls (27.5\% versus 44.4\%; $P<0.001)$, whereas the deletion of GSTT1 was found significantly more often in cases than in the controls $(49.3 \%$ versus $29.7 \% ; P<0.001$ ). We did not find statistically significant differences in the genotype distribution in GSTP1 (Ile105Val) polymorphism between cases and controls ( $40.5 \%$ versus $46.0 \%)$.

Conclusions: We may conclude that individuals homozygous for the deletion of GSTT1 are at an $\approx 2$-fold-greater risk of DR, whereas the GSTM1 deficiency is associated with lower frequency of DR in type 2 diabetics.
\end{abstract}

Keywords: Diabetes mellitus type 2, diabetic retinopathy, oxidative stress, glutathione S-transferase

\section{Introduction}

Type 2 diabetes mellitus is a common multifactorial genetic syndrome, which is determined by several different genes and environmental factors. It now affects 350 million people world wide but its incidence is in-

\footnotetext{
${ }^{1}$ The authors contributed equally to the manuscript.

* Corresponding author: Danijel Petrovič, MD, PhD, Institute of Histology and Embryology, Medical Faculty of Ljubljana, Korytkova 2, 1105 Ljubljana, Slovenia. Tel.: +386 1543 7367; Fax: +386 1 543 7361; E-mail: daniel.petrovic.@mf.uni-lj.si.
}

creasing rapidly because of secondary factors, such as obesity, hypertension, and lack of physical activity [16, 52]. Hyperglycaemia resulting from uncontrolled glucose regulation is widely recognized as a causal link between diabetes and diabetic complications, as it causes free radicals hyperproduction in endothelial cells at the mitochondrial level [11].

Substantial data indicate that oxidative stress is involved in the development of diabetic retinopathy (DR) $[3,22,30,33]$. Retina, a tissue rich in polyunsaturated fatty acid, uses more oxygen than any other tissue in the body, and is very susceptible to damage [34]. 
Oxidative stress is the result of an imbalance between the amount of reactive oxygen species (ROS) and the capacity of antioxidant defence systems [3].

Since long-term exposure to oxidative stress is strongly implicated in the pathogenesis of diabetic complications, polymorphic genes of detoxifying enzymes are implied in the development of DR. We assessed the potential role of glutathione S-transferase (GST) on the development of DR in patients with type 2 diabetes.

Glutathione S-transferases (GSTs) belong to a family of ubiquitous and multifunctional enzymes that work as one of the endogenous antioxidants in our body $[8$, 18].

GST enzymes are coded by at least eight distinct loci: $\alpha$ (GSTA), $\mu$ (GSTM), $\theta$ (GSTT), $\pi$ (GSTP), $\sigma$ (GSTS), $\kappa$ (GSTK), o (GSTO), and $\tau$ (GSTZ), each containing one or more homodimeric or heterodimeric isoforms. Three loci in particular, GSTM1, GSTT1, and GSTP1, have received most of the attention. The GSTM1 locus has been mapped on chromosome 1p13.3, while the GSTT1 and GSTP1 locus exist on chromosome 22q11.2 and 11q13. Persons with homozygous deletions of either the GSTM1 or GSTT1 locus have no enzymatic functional activity of the respective enzyme $[41,43]$.

A GSTP1 variant with a substitution in the active site of valine for isoleucine at codon 105 (Ile105Val) has a reduced ability to conjugate reactive electrophiles with glutathione and may therefore sensitize cells to free radical-mediated damage. The Val105 variant has been associated with susceptibility to smoking-related cancer and cardiovascular disease [21].

In contrast to the extensive investigation of the GSTM1 and GSTT1 gene variants in relation to malignancy $[9,31,35,41,46,59]$ there are few studies investigating the associations between GSTM1 and GSTT1 gene variants and macrovascular disease in diabetics $[21,28,40]$. Moreover, the association between the GSTM1 and GSTT1 gene variants and microvascular complications, namely DR and diabetic nephropathy, in type 2 diabetes has been evaluated only in two studies so far [17,21]. Moreover, recent studies have revealed significant interethnic differences in allelic frequencies of polymorphic GSTM1 and GSTT1 genes and susceptibility to certain diseases $[25,44]$.

The aim of the present study was to investigate whether the genetic polymorphisms: polymorphic deletions of glutathione S-transferases M1 (GSTM1) and T1 (GSTT1) and Ile105Val of the GSTP1 are associated with DR in a large sample of Caucasians with type 2 diabetes.

\section{Patients and methods}

In this cross-sectional case-control study 604 unrelated Caucasians with type 2 diabetes mellitus with a defined ophthalmologic status were enrolled (they have not been controlled for the glycemic history). Patients were classified as having type 2 diabetes according to the current American Diabetes Association criteria [26].

Fundus examination was performed by a senior ophthalmologist (M.P.) after pupil dilatation (tropicamide and phenylephrine $2.5 \%$ ) using slit lamp biomicroscopy with non-contact lens, and was electronically documented with a $50^{\circ}$-angle fundus camera (Topcon-TRC 40-IX; Tokyo, Japan). Staging of diabetic retinopathy was determined according to the ETDRS retinopathy severity scale [60].

The study group consisted of 604 subjects: 284 subjects with DR (cases) and the control group of 320 subjects with type 2 diabetes of more than 10 years' duration who had no clinical signs of diabetic retinopathy. The DR group consisted of 198 subjects with proliferative diabetic retinopathy (new vessel formation and/or fibrous proliferation with or without vitreous hemorrhage) and 86 subjects with non-proliferative diabetic retinopathy (microaneurysms, retinal hemorrhages, hard exudates) [60].

To avoid the confounding effect of impaired kidney function, the patients with overt nephropathy were not enrolled. The study was approved by the national medical ethics committee. After an informed consent for the participation in the study was obtained, a detailed interview was made.

Genomic DNA was extracted from $200 \mu$ l of whole blood using a FlexiGene DNA isolation kit according to the recommended protocol (Qiagene, Hilden; Germany).

Genotypes of GSTM1 and GSTT1 were determined by multiplex PCR amplification, as described previously [6]. For GSTT1-null/GSTM1-null genotype no bands were obtained, necessitating the use of albumin as internal positive control, in order to distinguish the null genotype from aborted PCR reactions. Amplification results in a 480 bp GSTT1 fragment, 215 bp GSTM1 fragment and $350 \mathrm{bp}$ albumin fragment [6]. By using this protocol it was not possible to distinguish homozygous and heterozygous carriers of GSTM and GSTT alleles [7].

The GSTP1 genotype was evaluated by PCR using the following primers: forward 5'- ACC CCA GGG CTC TAT GGG AA -3' and reverse 5'- TGA GGG 
Table 1

Characteristics of patients with diabetic retinopathy (cases) and patients without diabetic retinopathy (controls)

\begin{tabular}{lccc}
\hline Characteristics & Cases n $(\%)$ & Controls n $(\%)$ & $\mathrm{P}$ \\
\hline Number & 284 & 320 & \\
Age (years) & $65.6 \pm 9.6$ & $64.9 \pm 10.3$ & 0.4 \\
Male sex (\%) & $139(49)$ & $140(43.8)$ & 0.2 \\
Duration of diabetes (years) & $19.1 \pm 8.9$ & $11.9 \pm 8.1$ & $<0.001$ \\
Patients on insulin therapy (\%) & $201(70.8)$ & $119(37.3)$ & $<0.001$ \\
HbA $_{1 c}(\%)^{1}$ & $8.0 \pm 1.6$ & $7.7 \pm 1.6$ & 0.05 \\
Systolic blood pressure (mm Hg) $_{\text {Diastolic blood pressure (mm Hg) }}$ & $144 \pm 22$ & $143 \pm 19$ & 0.6 \\
BMI (kg/m $\left.{ }^{2}\right)$ & $28.3 \pm 4.7$ & $31.7 \pm 14.9$ & 0.01 \\
History of hypertension (\%) & $149(52.6)$ & $174(54.4)$ & 0.7 \\
Smokers $(\%)$ & $33(11.6)$ & $36(11.2)$ & 1.0 \\
Total cholesterol (mmol/l) & $4.9 \pm 1.3$ & $4.8 \pm 1.1$ & 0.8 \\
HDL cholesterol (mmol/l) & $1.2 \pm 0.3$ & $1.2 \pm 0.3$ & 0.96 \\
LDL cholesterol (mmol/l) & $2.7 \pm 0.9$ & $2.6 \pm 0.9$ & 0.4 \\
Triglycerides (mmol/l) & $2.9 \pm 3.1$ & $2.5 \pm 1.5$ & 0.4 \\
\hline
\end{tabular}

Data are expressed as means \pm standard deviations or frequencies (percentages).

${ }^{1}$ The average value of $\mathrm{HbA}_{1 c}$.

CAC AAG AAG CCC CT-3' as described previously [49]. The PCR product was then digested with $3 \mathrm{U}$ Alw261 (New England Biolabs, Hertfordshire, UK). In homozygotes for A allele a fragment of $176 \mathrm{bp}$ is seen. Restriction site appears when the $G$ allele is present and two fragments (91 bp+ $85 \mathrm{bp}$ ) are seen in homozygotes.

Genotyping was performed by two researchers (D.P., I.C.), blinded to the retinopathy status of the patients. Chi-square test was used to compare discrete variables. Continuous clinical data were compared by unpaired Students $t$ test. In addition, all variables that showed significant differences by univariate methods (chi-square test, unpaired Students $t$ test) were analysed together in a logistic regression analysis. A $p<$ 0.05 was considered statistically significant. Assuming the significance level of 0.05 , we calculated the power of our study sample (284 DR cases, 320 controls diabetics without DR) to be $99 \%$ for GSTT1-null and GSTM1-null genotypes.

Statistical analysis was performed using the SPSS program for Windows version 19 (SPSS Inc. Illinois).

\section{Results}

The characteristics of the cases and control subjects are summarized in Table 1. Cases had a longer duration of type 2 diabetes compared to the diabetics without DR. Additionally, they had a higher prevalence of insulin therapy than the controls (diabetics without DR). Body mass index (BMI) was higher in controls, but there were no significant differences in hypertension, smoking, LDL and HDL cholesterol levels and triglyceride levels between the cases and controls.

The GSTP1 codon 105 variant was present in HardyWeinberg equilibrium (HWE) (GSTP1 cases: ${ }^{2}=$ $0.856, p=0.35$; GSTP1 controls: ${ }^{2}=0.15, p=$ 0.7); however, HWE was not assessed for the GSTM1 and GSTT1 variants because heterozygous individuals could not be distinguished from the homozygous wild type. The distribution of GSTT1, GSTM1 and GSTP1 genetic polymorphisms among patients with DR (cases) and patients without DR (controls) are shown in Table 2. A significantly higher frequency of the GSTT1-null genotype was found in cases in comparison with controls (diabetics without DR). Moreover, the GSTM1-null genotype was significantly less frequent in controls.

Univariate analysis showed that the following variables were associated with the DR: GSTM1-null genotype, GSTT1-null genotype, duration of diabetes, patients on insulin therapy and BMI.

The variables showing an association $(p<0.05)$ were then put into a stepwise multiple logistic regression in order to study the possible effect of the GSTT1null and GSTM1-null with other risk factors on DR development.

In the study, we found out that the absence of the GSTT gene (GSTT1-null genotype) was associated with an increased risk of DR. Contrary, the GSTM1null genotype conferred a reduced risk of DR (Table 2). There was no evidence that GSTP1 variants were associated with DR in this population (Table 2).

After the adjustment for BMI, the duration of diabetes, insulin therapy and age, the carriers of GSTT1- 
Table 2

Distribution of different genetic polymorphisms amongst subjects with DR (cases) and amongst diabetics without DR (controls)

\begin{tabular}{|c|c|c|c|c|c|c|}
\hline & \multicolumn{2}{|c|}{ Cases (284) } & \multicolumn{2}{|c|}{ Controls (320) } & \multirow[t]{2}{*}{ OR $(95 \% \mathrm{CI})^{1}$} & \multirow[t]{2}{*}{$\mathrm{P}$} \\
\hline & No. & $\%$ & No. & $\%$ & & \\
\hline \multicolumn{7}{|l|}{ GSTP1 } \\
\hline Genotype AA & 115 & 40.5 & 147 & 46.0 & $0.863(0.628-1.187)$ & 0.373 \\
\hline Genotype AG & 137 & 44.4 & 142 & 44.4 & $1.252(0.908-1.725)$ & 0.192 \\
\hline Genotype GG & 32 & 11.4 & 31 & 9.6 & Reference & \\
\hline \multicolumn{7}{|l|}{ GSTM1 } \\
\hline GSTM1-null & 78 & 27.5 & 142 & 44.4 & $0.475(0.337-0.668)$ & $<0.001$ \\
\hline GSTM1-present & 206 & 72.5 & 178 & 55.6 & Reference & \\
\hline \multicolumn{7}{|l|}{ GSTT1 } \\
\hline GSTT1-null & 140 & 49.3 & 95 & 29.7 & $2.303(1.649-3.216)$ & $<0.001$ \\
\hline GSTT1-present & 144 & 50.7 & 225 & 70.3 & Reference & \\
\hline
\end{tabular}

${ }^{1} \mathrm{OR}$, odds ratio; CI, confidence interval. The data were analyzed by the $\chi^{2}$ test.

Table 3

Adjusted odds ratios for risk factors for diabetic retinopathy according to multiple logistic regression analysis

\begin{tabular}{lcc}
\hline Risk factors & OR $(95 \%$ CI $)$ & P \\
\hline GSTT1-null & $2.167(1.437-3.267)$ & $<0.001$ \\
GSTM1-null & $0.589(0.387-0.896)$ & 0.013 \\
Duration of diabetes & $0.899(0.874-0.924)$ & $<0.001$ \\
BMI & $1.068(1.021-1.068)$ & 0.004 \\
Insulin therapy & $2.422(1.583-3.705)$ & $<0.001$ \\
Age & $1.044(1.021-1.068)$ & $<0.001$ \\
\hline
\end{tabular}

null genotype showed a 2.167-fold higher risk for DR $(\mathrm{OR}=2.167 ; 95 \% \mathrm{CI}=1.437-3.267 ; p<0.001$; Table 3). Carriers of GSTM1-null genotype showed as much as a $61.3 \%$ reduction in relative risk or as little as a $10.4 \%$ reduction in risk $(\mathrm{OR}=0.589 ; 95 \% \mathrm{CI}=$ 0.387-0.896; $p=0.013$; Table 3).

\section{Discussion}

As GSTM1 and GSTT1 are involved in the processing of reactive oxygen, lipid peroxidation products and some key metabolites of toxicants, there are potential links between genetic polymorphisms of these enzymes and the pathogenesis of a number of chronic diseases. In particular, important insights into the effects of the GSTM1 and GSTT1 gene deletions on the pathogenesis of human diseases have been derived from molecular epidemiological studies [10]. To better understand the role of GST genotypes on the development of DR, the aforementioned loci were selected for the evaluation in a large sample of Caucasians with type 2 diabetes.

In our study, the GSTT1-null genotype was found to be statistically significantly more frequent in the cases with DR compared to the controls (49.3\% vs. $29.7 \%$; $p<0.001$ ) suggesting the GSTT1-null genotype to the genetic risk factor for DR in subjects with type $2 \mathrm{di}-$ abetes. Our results are not in accordance with the results of the study in Slovenian Caucasians with type 1 diabetes [29]. In the study in children with type 1 diabetes, the prevalence of GSTT1-null genotype was $18.8 \%$ among patients with DR and $25 \%$ among diabetics without DR [29]. Our findings, on the other hand, are in agreement with reports by Datta et al. [17] and Doney et al. [21], who have recently reported the GSTT1-null genotype to be associated with diabetic nephropathy. Moreover, it was also reported that individuals homozygous for the GSTT1-null allele had a more generalized vasculopathy, with an increased risk of progression of both diabetic nephropathy and sightthreatening retinopathy, and this association was not influenced by smoking status [21].

In the study we demonstrate a lower frequency of the GSTM1 gene deletion polymorphism in DR in comparison to diabetics without DR (27.5\% vs. $44.4 \%)$. We report for the first time that the GSTM1 deficiency might confer protection against the development of DR in people with type 2 diabetes. Moreover, the association between the GSTM1-null genotype and DR remained significant after adjusting for age, duration of diabetes, insulin therapy and BMI. To our knowledge, only one study [29] revealed similar evidence of association between the GSTM1-null genotype and the reduced risk of DR in a smaller prospective study in type 1 diabetes.

There are various reports on the association between GSTM1-null genotype and reduced risk for some cancers [36,38,57]. Lin et al. [37] found that people who are deficient in GSTM1 and who consume broccoli are protected against colon cancer, due to the potentially slower excretion of isothiocyanates. The protecting effect of isothiocyanates against lung cancer was shown also in individuals consuming cruciferous vegetables 
and bearing the GSTM1-null genotype [38]. Furthermore, studies by Xiao and Singh [56] provide support for isothiocyanate-mediated inhibition of angiogenesis. They showed that isothiocyanate supress expression of nuclear factor- $\kappa \mathrm{B}$-regulated genes, including vascular endothelial growth factor (VEGF), a primary suspect in induction of retinal neovascularization in diabetes $[1$, $4,58]$.

Independent of the genetic factors, insulin therapy was statistically significantly associated with DR. This finding suggests the existence of other factors, such as differences in the ability of insulin secretion, or differences in the frequency of episodes of hypoglycemia, or adverse events associated with insulin therapy (hypoglycemia, worsening diabetic retinopathy if $\mathrm{HbA}_{1 c}$ decreases rapidly) [20,45]. Logistic regression analysis demonstrated that beside genetic factors (GSTT1-null and GSTM1-null genotypes) and insulin therapy, the duration of diabetes was an independent risk factor for DR. The duration of diabetes [23,53], high BMI [32,47] and glycaemic control [12,51] are the most important factors associated with the development of retinopathy. Today, $\mathrm{HbA}_{1 c}$ measurement is regarded as the "gold standard" indicator for glycaemic control in diabetic patients, reflecting glucose levels over $2-3$ month period $[5,42]$.

Large ethnic differences in the prevalence of the homozygously deleted GSTT1 genotype have been observed. The prevalence of the GSTT1-null genotype is highest among Chinese (64\%), followed by Koreans (60\%), African-Americans (22\%), Caucasians (29\%) and Asian-Indians (16\%), whereas it is lowest among Mexian-Americans (10\%) [61,62]. In the present study, the GSTT1-null genotype frequency found in the control group $(29.7 \%)$ did not vary very much from already known Slovenian frequency $(25.5 \%)$ and from European and Mediterranean ones (from 10.4 to $42.5 \%$ ) [25]. Similarly, the GSTM1 is polymorphic, and is absent in 10-65\% of the human population [63]. In our study, the prevalence of the GSTM1-null genotype was $44.4 \%$ among the controls, and fits within the range of variability observed in the Mediterranean basin and in Europe [25,44].

It is now generally accepted that retinopathy is a low-grade chronic inflammatory condition associated with increased leukocyte entrapment in retinal capillaries, and areas of capillary non-perfusion and endothelial cell damage [2]. Apart from realising enhanced amounts of ROS when compared with normal cells $[14,19,48, ?]$, leukocytes from diabetic patients also synthesise leukotriene B4 [27,50], which in turn may act along with other inflammatory mediators including prostaglandins [13] to promote changes in retinal vasculature. Since some members of GSTs are involved in the biosynthesis of prostaglandins and leukotriens [15] the absence of enzyme activity of GSTM1 might be protective against DR in type 2 diabetes.

Type 2 diabetes is strongly associated with atherosclerosis and it represents a major risk factor for cardiovascular disease (CAD). Despite the prominent role of oxidative stress in the development of CAD, few studies have indicated that GSTM1-null genotype is protective against $\mathrm{CAD}[54,55]$. The up-regulation of another enzyme more effective at detoxification of atherogenic compounds and higher activity of CYP1A2 in individuals null for GSTM1 [39] might offer another reasonable explanation for the protective effect of GSTM1-null genotype.

However, it should be stressed that to date we do not have any firm evidence that such mechanisms promote efficient defence against the development and progression of DR in type 2 diabetics. Hence, additional research is mandatory for answering this question.

\section{Conclusions}

We may conclude that individuals homozygous for the deletion of GSTT1 are at an $\approx 2$-fold-greater risk of DR, whereas the GSTM1 deficiency is associated with lower frequency of DR in type 2 diabetics. Finally, further research is required to clarify potential protective role of GSTM1 null genotype against DR in type 2 diabetes.

\section{Acknowledgement}

The authors thank Mrs. Visam Bajt, BA, for revising the English.

\section{References}

[1] L.P. Aiello, S.E. Brusell, A. Clermont, E. Duh, H. Ishii, C. Takagi, F. Mori, T.A. Ciulla, K. Ways, M. Jirousek, L.E. Smith and G.L. King, Vascular endothelial growth factor-induced retinal permeability is mediated by protein kinase $\mathrm{C}$ in vivo and suppressed by an orally effective beta-isoform-selective inhibitor, Diabetes 46 (1997), 1473-1480.

[2] A.P. Adamis, Acute intensive insulin therapy exacerbates diabetic blood-retinal barrier breakdown via hypoxia-inducible factor-1 and VEGF, J Clin Invest 109 (2002), 805-815.

[3] A.G. Allen and M. Tresini, Oxidative stress and gene regulation, Free Radic Biol Med 28 (2000), 463-499. 
[4] M. Amadio, G. Scapagnini, G. Lupo, F. Drago, S. Govoni and A. Pascale, PKCbetaII/HuR/VEGF: a new molecular cascade in retinal pericytes for the regulation of VEGF gene expression, Pharmacol Res 57 (2008), 60-66.

[5] American Diabetes Association, Postprandial blood glucose, Diabetes Care 24 (2001), 775-778.

[6] M. Arand, R. Mühlbauer, J. Hengstler, E. Jäger, J. Fuchs, L. Winkler and F. Oesch, A multiplex polymerase chain reaction protocol for the simultaneous analysis of the glutathione S-transferase GSTM1 and GSTT1 polymorphisms, Anal Biochem 236 (1996), 184-186.

[7] S.W. Baxter, E.J. Thomas and I.G. Campbell, GSTM1 null polymorphism and susceptibility to endometriosis and ovarian cancer, Carcinogenesis 22 (2001), 63-65.

[8] B. Blanchette, X. Feng and B.R. Singh, Marine glutathione S-transferases, Mar biotechnol 9 (2007), 513-542.

[9] S. Boccia, G. La Torre, F. Gianfagna, A. Mannocci and G. Ricciardi, Glutathione S-transferase T1 status and gastric cancer risk: A meta-analysis of the literature, Mutagenesis 21 (2006), 115-123.

[10] H.M. Bolt and R. Thier, Relevance of the deletion polymorphisms of the glutathione S-transferases GSTT1 and GSTM1 in pharmacology and toxicology, Curr Drug Metab 7 (2006), 613-628.

[11] M. Brownlee, Biochemistry and molecular cell biology of diabetic complications, Nature 414 (2001), 813-820.

[12] G. Cahill, L. Etzweiler and N. Freinkel, Control and diabetes, N Engl J Med 294 (1976), 1004-1005.

[13] J. Cai and M. Boulton, The pathogenesis of diabetic retinopathy: old concepts and new questions, Eye 16 (2002), 242-260.

[14] M. Cantero, T. Parra and J.R. Conejo, Increased hydrogen peroxide formation in polymorphonuclear leukocytes of IDDM patients, Diabetes Care 21 (1998), 326-327.

[15] E.G. Chronopoulou and N.E. Labrou, Glutathione transferases: emerging multidisciplinary tools in red and green biotechnology, Recent Pat Biotecnol 3 (2009), 211-223.

[16] G. Danaei, M.M. Finucane, Y. Lu, G.M. Singh, M.J. Cowan, C.J. Paciorek, J.K. Lin, F. Farzadfar, Y.H. Khang, G.A. Stevens, M. Rao, M.K. Ali, L.M. Riley, C.A. Robinson and M. Ezzati, National, regional, and global trends in fasting plasma glucose and diabetes prevalence since 1980: systematic analysis of health examination surveys and epidemiological studies with 370 country-years and 2.7 million participants, Lancet 378 (2011), 31-40.

[17] S.K. Datta, V. Kumar, R.S. Ahmed, A.K. Tripathi, O.P. Kalra and B.D. Banerjee, Effect of GSTM1 and GSTT1 double deletions in the development of oxidative stress in diabetic nephropathy patients, Indian J Biochem Biophys 47 (2010), $100-103$.

[18] S.K. Datta, V. Kumar, R. Pathak, A.K. Tripathi, R.S. Ahmed, O.P. Kalra and B.D. Banerjee, Association of glutathione Stransferase M1 and T1 gene polymorphism with oxidative stress in diabetic and nondiabetic chronic kidney disease, Ren Fail 32 (2010), 1189-1195.

[19] S. Devaraj and I. Jialal, Low-density lipoprotein postsecretory modification, monocyte function, and circulating adhesion molecules in type 2 diabetic patients with and without macrocvascular complications: the effect of alpha-tocopherol supplementation, Circulation 102 (2000), 191-196.

[20] D.E. DeWitt and I.B. Hirsch, Outpatient insulin therapy in type 1 and type 2 diabetes mellitus: scientific review, JAMA 289 (2003), 2254-2264.

[21] A.S. Doney, S. Lee, G.P. Leese, A.D. Morris and C.N. Palmer, Increased cardiovascular morbidity and mortality in type 2 diabetes is associated with the glutathione $\mathrm{S}$ transferase thetanull genotype: a Go-DARTS study, Circulation 111 (2005), 2927-2934.

[22] Y. Du, C.M. Miller and T.S. Kern, Hyperglycemia increases mitochondrial superoxide in retina and retinal cells, Free Radical Biol Med 35 (2003), 1491-1499.

[23] D.S. Fong, L. Aiello, T.W. Gardner, G.L. King, G. Blankenship, J.D. Cavallerano, F.L. Ferris and R. Klein, Retinopathy in diabetes, Diabetes Care 27(Suppl 1) (2004), 584-587.

[24] S. Freedman and D. Hatchell, Enhanced superoxide radical production by stimulated polymorphonuclear leukocytes in a cat model of diabetes, Exp Eye Res $\mathbf{5 5}$ (1992), 767-773.

[25] S. Garte, L. Gaspari, A.-K. Alexandrie et al., Metabolic gene polymorphism frequencies in controll populations, Cancer Epidemiol Biomarkers Prev 10 (2001), 1239-1248.

[26] S. Genuth, K. G. Alberti, P. Bennett, J. Buse, R. Defronzo, R. Kahn, J. Kitzmiller, W.C. Knowler, H. Lebovitz, A. Lernmark, D. Nathan, J. Palmer, R. Rizza, C. Saudek, J. Shaw, M. Steffes, M. Stren, J. Tuomilehto and P. Zimmet, Expert Commitee on the Diagnosis and Classification of Diabetes Mellitus, Followup report on the diagnosis of diabetes mellitus, Diabetes Care 26 (2003), 3160-3167.

[27] R.A. Gubitosi-Klug, R. Talahalli, Y. Du, J.L. Nadler and T.S. Kern, 5-Lipoxygenase, but not 12/15-lipoxygenase, contributes to degeneration of retinal capillaries in a mouse model of diabetic retinopathy, Diabetes 57 (2008), 1387-1393.

[28] A.M. Hossaini, I.M. Zamrroni, R.A. Kashem and Z.F. Khan, Polymorphism of glutathione S-transferases as genetic risk factors for the development of complications in type 2 diabetes mellitus, J Crit Care 23 (2008), 444-448.

[29] T. Hovnik, V. Dolžan, N. Uršič Bratina, K. Trebušak Podkrajšek and T. Battelino, Genetic polymorphisms in genes encoding antioxidant enzymes are assoviated with diabetic retinopathy in type 1 diabetes, Diabetes Care 32 (2009), 22582262.

[30] S.K. Jain, R. McVie, J. Duett and J.J. Herbst, Erythrocyte membrane lipid peroxidation and glycosylated hemoglobin in diabetes, Diabetes 38 (1989), 1539-1543.

[31] T. Katoh, Y. Yamano, M. Tsuji and M. Watanabe, Genetic polymorphisms of human cytosol glutathione S-transferases and prostate cancer, Pharmacogenomis 9 (2008), 93-104.

[32] M.W. Knuiman, T.A. Welborn, V.J. McCann, K.G. Stanton and I.J. Constable, Prevalence of diabetic complications in relation to risk factors, Diabetes 35 (1986), 1332-1339.

[33] R.A. Kowluru, L. Atasi and Y.S. Ho, Role of Mitochondrial Superoxide Dismutase in the Development of Diabetic Retinopathy, Invest Ophthalmol, Vis Sci 47 (2006), 15941599.

[34] R.A. Kowluru, V. Kowluru, Y. Xiong and Y.S. Ho, Overexpression of mitochondrial superoxide dismutase in mice protects the retina from diabetes-induced oxidative stress, Free Radic Biol Med 41 (2006), 1191-1196.

[35] S. Landi, Mammalian class theta GST and differential susceptibility to carcinogens: A review, Mutat Res 463 (2000), 247-283.

[36] S.J. Lewis, N.M. Cherry, R.M. Niven, P.V. Barber and A.C. Povey, GSTM1, GSTT1 and GSTP1 polymorphisms and lung cancer risk, Cancer Lett 180 (2002), 165-171.

[37] H.J. Lin, N.M. Probst-Hensch, A.D. Louie, I.H. Kau, J.S. Witte, S.A. Ingles, H.D. Frankl, E.R. Lee and R.W. Haile, Glutathione transferase null genotype, broccoli, and lower prevalence of colorectal adenomas, Cancer Epidemiol Biomarkers Prev 7 (1998), 647-652. 
[38] S.J. London, J.M. Yuan, F.L. Chung, Y.T. Gao, G.A. Coetzee, R.K. Ross and M.C. Yu, Isothiocyanates, glutathione Stransferase $\mathrm{M} 1$ and $\mathrm{T} 1$ polymorphisms, and lung-cancer risk: a prospective study of men in Shanghai, China, Lancet $\mathbf{3 5 6}$ (2000), 724-729.

[39] S. MacLeod, R. Sinha, F.F. Kadlubar and N. Lang, Polymorphisms of CYP1a1 and GSTM1 influence the in vivo function of CYP1A2, Mutat Res 376 (1997), 135-142.

[40] S. Manfredi, D. Calvi, M. del Fiandra, N. Botto, A. Biagini and M.G. Andreassi, Glutathione S-transferase T1- and M1null genotypes and coronary artery disease risk in patients with Type 2 diabetes mellitus, Pharmacogenomics 10 (2009), 29-34.

[41] Z. Mo, Y. Gao, Y. Cao, F. Gao and L. Jian, An updating metaanalysis of the GSTM1, GSTT1, and GSTP1 polymorphisms and prostate cancer: a HuGE review, Prostate 69 (2009), 662 688.

[42] B. Ozmen and S. Boyvada, The relationship between self-monitoring of blood glucose control and glycosylated haemoglobin in patients with type 2 diabetes with and without diabetic retinopathy, J Diabtes Complications 17 (2003), 128-134.

[43] S. Pemble, K.R. Schroeder, S.R. Spencer, D.J. Meyer, E. Hallier, H.M. Bolt, B. Ketterer and J.B. Taylor, Human gluthatione S-transfrase Theta (GSTT1): cDNA cloning and the characterization of a genetic polymorphism, Biochem J 300 (1994), 271-276.

[44] S. Piacentini, R. Polimanti, F. Porreca, C. Martínez-Labarga, G. F. De Stefano and M. Fuciarelli, GSTT1 and GSTM1 gene polymorphisms in European and African populations, Mol Biol Rep 38 (2011), 1225-1230.

[45] V Poulaki, W. Qin, A.M. Joussen, P. Hurlbut, S.J. Weigand, J. Rudge, G.D. Yancopoulos and A.P. Adamis, Acute intensive insulin therapy exacerbates diabetic blood-retinal barrier breakdown via hypoxia-inducible factor- $1 \alpha$ and VEGF, $J$ Clin Invest 109 (2002), 805-815.

[46] T.R. Rebbeck, Molecular epidemiology of the human glutathione S-transferase genotypes GSTM1 and GSTT1 in cancer susceptibility, Cancer Epidemiol Biomarkers Prev 6 (1997), 733-743.

[47] K.G. Santos, J.R. Tschiedel, K.E.P. Scheneider and I. Roisenberg, Prevalence of retinopathy in Caucasian type 2 diabetic patients from South Brazil and relationship with clinical and metabolic factors, Braz J Med Biol Res 38 (2005), 221-225.

[48] S.V. Shah, J.D. Vallin and S.D. Eilen, Chemiluminescence and superoxide anion production by leukocytes from diabetic patients, J Clin Endocrinol Metab 57 (1983), 402-409.

[49] K. Sundberg, A.S. Johansson, G. Stenberg, M. Widersten, A. Seidel, B. Mannervik and B. Jernström, Differences in the catalytic efficiencies of allelic variants of glutathione transferase P1-1 towards carcinogenic diol epoxides of polycyclic aromatic hydrocarbons, Carcinogenesis 19 (1998), 433-436.

[50] R. Talahalli, S. Zarini, N.S. Sheibani, R.C. Murphy and R.A. Gubitosi-King, Increased synthesis of leukotrienes in the mouse model of diabetic retinopathy, Invest Ophtalmol Vis Sci 51 (2010), 1699-1708.
[51] The Diabetes Control and Complications Trial Research Group, The effect of intensive insulin treatment of diabetes on the development and progression of long term complications in insulin dependent diabetes mellitus, $N$ Engl J Med 329 (1993), 977-986.

[52] J. van Tilburg, T.W. van Haeften, P. Pearson and C. Wijmenga, Defining the genetic contribution of type 2 diabetes mellitus, J Med Genet 38 (2001), 569-578.

[53] S. Vinker, M. Shpiz, A. Elhayany and S. Nakar, Improvement of early detection of diabetic retinopathy-a primary care intervention study, Harefuah 142 (2003), 826-828.

[54] M.H. Wilson, P.J. Grant, L.J. Hardie and C.P. Wild, Gluatthione S-transferase M1 null genotype is associated with decreased risk of myocardial infarction, FASEB $J \mathbf{1 4}$ (2000), 791-796.

[55] M.H. Wilson, P.J. Grant, K. Kain, D.P. Warner and C.P. Wild, Association between the risk of coronary artery disease in South Asians and a deletion polymorphism in glutathione Stransferase M1, Biomarkers 8 (2003), 43-50.

[56] D. Xiao and S.V. Singh, Phenethyl isothiocynate inhibits angiogenesis in vitro and ex vivo, Cancer Res 67 (2007), 22392246.

[57] D.S. Yadav, T.R. Devi, R. Ihsan, A.K. Mishra, M. Kaushal, P.S. Chauhan, S.A. Bagadi, J. Sharma, E. Zamoawia, Y. Verma, A. Nandkumar, S. Saxena and S. Kapur, Polymorphisms of glutathione-S-transferase genes and the risk of aerodigestive tract cancers in the Northeast Indian population, Genet test Mol Biomarkers 14 (2010), 715-723.

[58] T. Yokota, R.C. Ma, J.Y. Park, K. Isshiki, K.B. Sotiropoulos, R.K. Rauniyar, K.E. Bornfeldt and G.L. King, Role of protein kinase $\mathrm{C}$ on the expression of platelet-derived growth factor and endothelin-1 in the retina od diabetic rats and cultured retinal capillary perycites, Diabetes $\mathbf{5 2}$ (2003), 838-845.

[59] S. Zhong, A. Howie, B. Ketterer, C.R. Wolf and N.K. Spurr, Glutathione S-transferases mu locus: Use of genotyping and phenotyping assays to assess association with lung cancer susceptibility, Carcinogenesis 12 (1991), 1533-1537.

[60] Early Treatment Diabetic Retinopathy Study Research Group: Grading diabetic retinopathy from stereoscopic color fundus photographs-an extension of the modified Airlie House classification ETDRS report number 10, Ophthalmology $\mathbf{9 8}$ (Suppl 5) (1991), 786-806.

[61] E.J. Lee, J.Y. Wong, P.N. Yeoh and N.H. Gong, Glutathione S-transferase theta (GST1) genetic polymorphism among Chinese, Malays and Indians in Singapore, Pharmacogenetics 5 (1995), 332-334.

[62] H.H. Nelson, J.K. Wiencke, D.C. Christiani, T.J. Cheng, Z.F. Zuo, B.S. Schwartz, B.K. Lee, M.R. Spitz, M. Wang and X. $\mathrm{Xu}$, Etnic diffrences in the prevalence of the homozygously deletd genotype of glutathione S-transferase theta, Carcinogenesis 16 (1995), 1243-1245.

[63] S.C. Choi, K.J. Yun, T.H. Kim et al., Prognostic potential of glutathione S-transferase M1 and T1 null genotypes for gastric cancer progression, Cancer Lett 195 (2003), 169-175. 


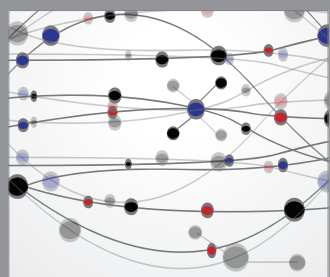

The Scientific World Journal
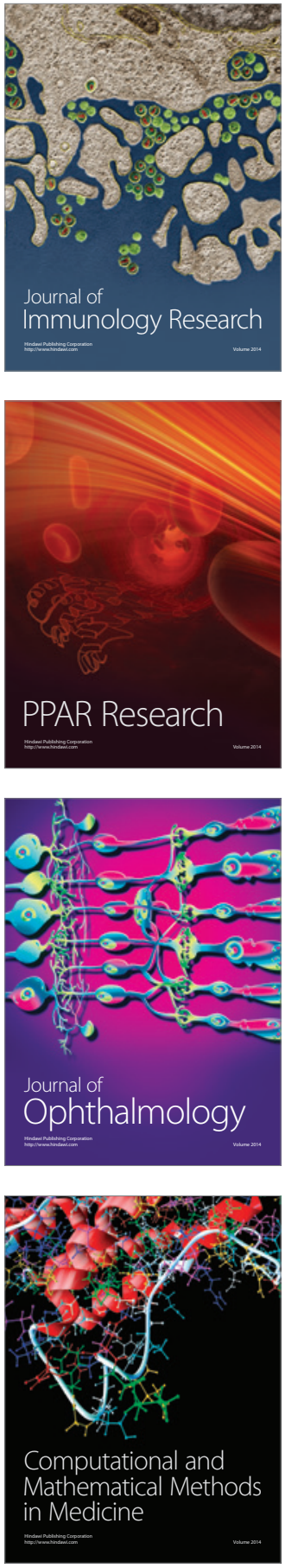

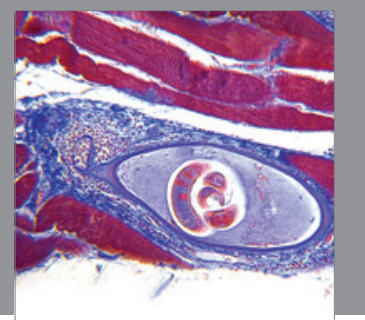

Gastroenterology

Research and Practice
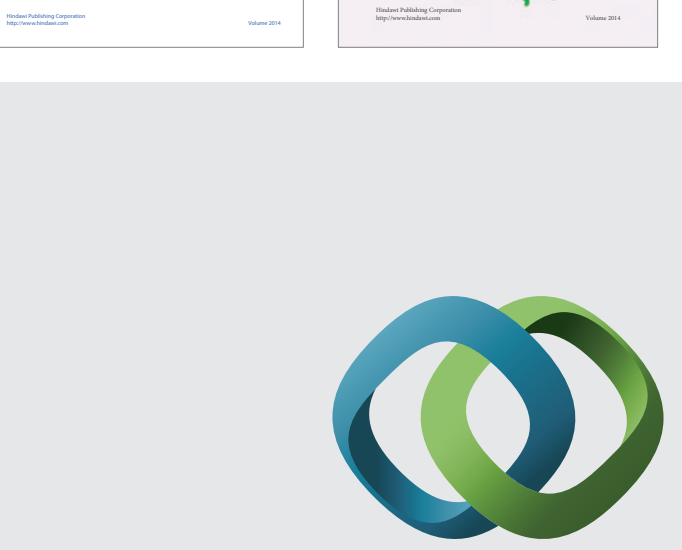

\section{Hindawi}

Submit your manuscripts at

http://www.hindawi.com
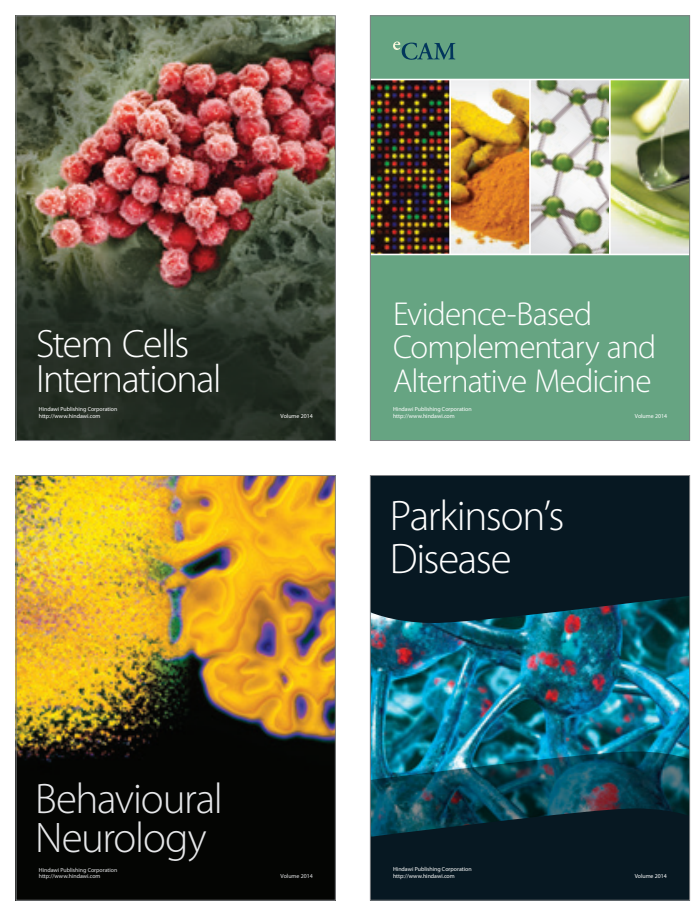

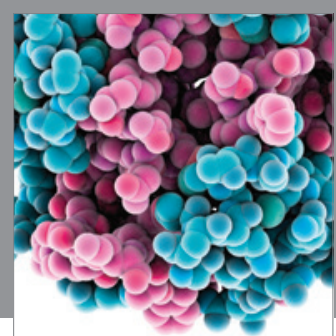

Journal of
Diabetes Research

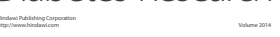

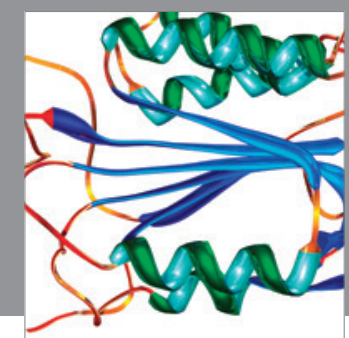

Disease Markers
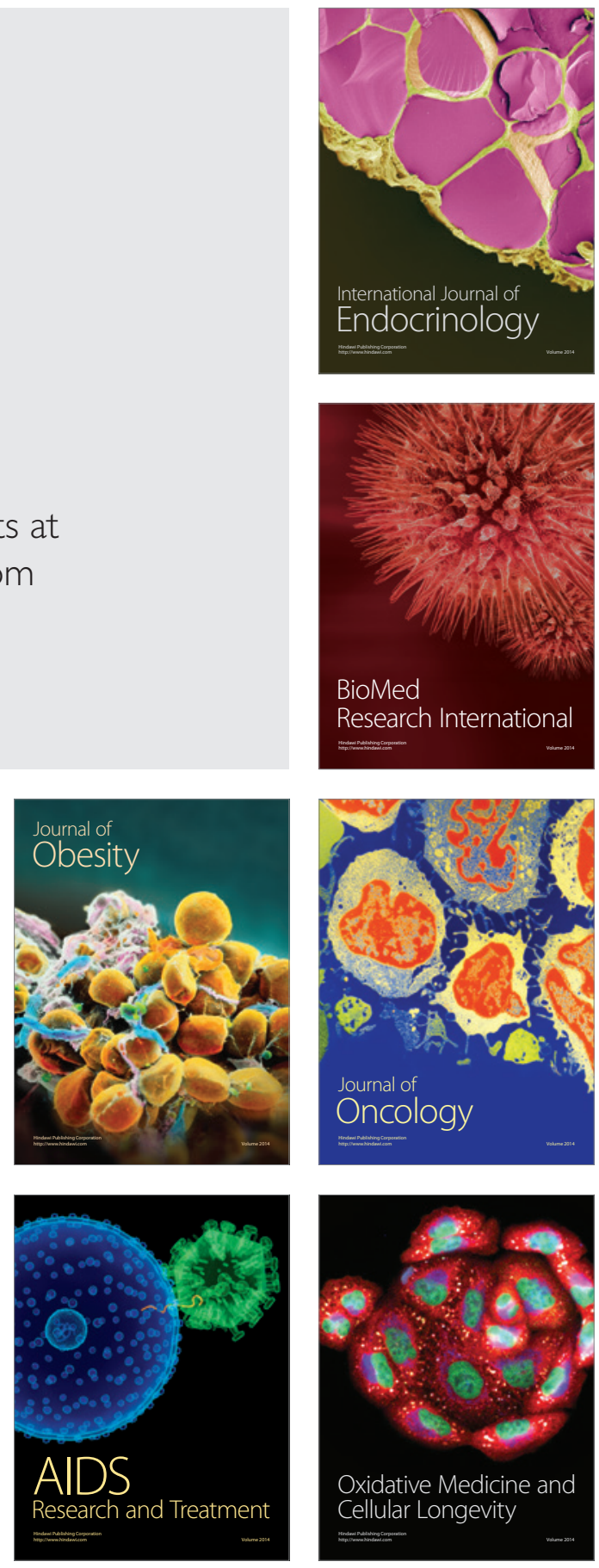